\title{
LOS ALUDES DE NIEVE EN EL ALTO SIL (OESTE DE LA CORDILLERA CANTÁBRICA, ESPAÑA)
}

\author{
J. SANTOS GONZÁLEZ, J. M. REDONDO VEGA, \\ A. GÓMEZ VILLAR y R. B. GONZÁLEZ GUTIÉRREZ \\ Departamento de Geografía y Geología \\ Universidad de León. Campus de Vegazana, s/n. 24071 León \\ Correo electrónico de contacto: jsango@unileon.es
}

\begin{abstract}
RESUMEN. Se presenta una cartografía de detalle de riesgo de aludes para el Alto Sil, la primera de estas características en la Cordillera Cantábrica. Su realización está basada en observaciones de campo, en encuestas a población local y en la utilización de un Sistema de Información Geográfica. A partir de una primera aproximación en la que se definen las zonas con pendientes, orientaciones, altitudes y vegetación adecuadas para el desencadenamiento de aludes, se ha realizado una cartografía detallada que ha permitido definir las zonas afectadas por aludes recientes, las zonas de riesgo elevado de avalanchas y las zonas de riesgo bajo. De todo ello se presentan dos ejemplos para los entornos de Valseco y Villarino del Sil, donde los aludes tienen mayor trascendencia actualmente.
\end{abstract}

ABSTRACT. For the first time in a sector of the Cantabrian Mountains, the Alto Sil, a detailed avalanche hazard cartography has been carried out. This cartography is based in field observations, enquiries to local people and the use of a Geographic Information System. In a first approach we define areas with suitable slopes, orientations, altitudes and vegetation cover for avalanches. Therefore, we present maps with recent, high risk and low risk avalanche areas. Finally, two maps are shown as an example of zones where avalanches are more relevant, the Valseco and Villarino del Sil areas.

Palabras clave: Cordillera Cantábrica, riesgos naturales, aludes, SIG.

Key words: Cantabrian Mountains, natural hazards, avalanches, GIS. 


\section{Introducción}

Los aludes, o avalanchas de nieve, se definen como desprendimientos de nieve que se deslizan ladera abajo a partir de un punto o línea de debilidad (Carcavilla y López, 2000). Aunque en zonas de la montaña central y oriental leonesa estos deslizamientos de nieve se denominan nades y mueldas, respectivamente (Fernández Miguélez, 2000), en el Alto Sil, al igual que en el occidente de Asturias, son conocidos como ádenes.

En función del tipo de nieve desplazada, las formas de rotura, el trazado del alud o la superficie de deslizamiento se han establecido diversas clasificaciones (Carcavilla y López, 2000; Furdada, 2006), siendo las avalanchas de fondo y / o de nieve húmeda las que presentan un mayor potencial morfogenético, ya que es habitual que incorporen cantos, bloques o árboles en su recorrido (Decaulne y Sæmundsson, 2006).

Para que se desencadenen aludes, la pendiente de la ladera tiene que estar comprendida entre $28^{\circ}$ y $45^{\circ}$, influyendo también la forma de la pendiente, la rugosidad y la vegetación (Julián et al., 2000). En cualquier caso, los ádenes suelen producirse sobre todo en las denominadas zonas de aludes, superficies topográficas sobre las que se producen de forma periódica, teniendo una zona de salida, una zona de trayecto y una zona de llegada (Mases, 2005).

En España, entre 1970 y 1999, hubo 105 muertos por avalanchas, 10 de ellos en la Cordillera Cantábrica (Carcavilla y López, 2000), habiéndose incrementado el número de fallecidos en los últimos años debido, sobre todo, a un mayor uso de la montaña en los meses invernales (Martín, 2003). En los Pirineos, donde son más frecuentes y mejor conocidas, hay un seguimiento continuo para establecer las zonas de mayor riesgo, así como cartografías específicas (Mases y Vilaplana, 1991; Furdada, 1996; Julián y Chueca, 1999; Julián et al., 2000; Oller et al., 2006; Gavaldà y Moner, 2008). En el caso de Cataluña, la cartografía está disponible en la página web del Institut Cartogràfic de Catalunya (http://www.icc.es).

Sin embargo, en la Cordillera Cantábrica no existe cartografía de riesgo de aludes, habiéndose dado únicamente los primeros pasos en este sentido, con el establecimiento de zonas de riesgo a nivel regional (Marquínez et al., 2003; Wozniak y Marquínez, 2004). Esta situación contrasta con la amenaza que suponen los aludes en algunos sectores de la Cordillera, como en Peña Ubiña (Castañón, 1984), en el Puerto de San Isidro o, especialmente, en Picos de Europa (González Trueba, 2006), donde son frecuentes y de dimensiones importantes, provocando daños tanto en las infraestructuras como en la cubierta forestal. Además, en cuanto a aludes históricos, pueden destacarse los 19 muertos que se produjeron en varias localidades de Asturias en 1888, 11 de ellos en un solo evento en Pajares o los 6 muertos en Huelde, en 1931 (Alcalde, 2002).

En el Alto Sil, aunque en los últimos años no ha habido víctimas mortales por aludes, las condiciones topográficas y climáticas son favorables para su desarrollo en determinadas zonas, habiendo causado daños a infraestructuras recientemente. Todo esto justifica la 
necesidad de realizar una cartografía específica de aquellas zonas potencialmente expuestas a este fenómeno (Cruz, 1987), que sirva como herramienta para la ordenación del territorio, sobre todo si tenemos en cuenta el creciente uso turístico de estas montañas.

\section{2. Área de estudio}

El Alto Sil se encuentra en la vertiente meridional del occidente de la Cordillera Cantábrica, al noroeste de la provincia de León. Se trata de una zona de fuertes desniveles, con altitudes comprendidas entre los $2.136 \mathrm{~m}$ de altitud (Pico Valdeiglesia) y los 760 m (desembocadura del río Valdeprado en el río Sil). El río Sil articula este espacio discurriendo de NE a SW y conectando una serie de pequeños valles transversales (Fig. 1).

Por la margen derecha, los valles de Valdeprado, Pedroso, Palacios, Fuexo, Tejedo y Villarino salvan los desniveles que separan los 1.800-2.000 m de las cumbres y los $760-900 \mathrm{~m}$ del fondo del valle en distancias de entre 5 y $14 \mathrm{~km}$. Por la margen izquierda, los pequeños valles de Cuvachín, Reguera Durria, La Seita y Matalavilla salvan ese mismo desnivel en apenas 3-5 km. Por último, los valles de Valseco y Salentinos, con 21 y $14 \mathrm{~km}$ respectivamente, son las arterias principales de esta zona y en ellos se concentran las cumbres más elevadas, que sobrepasan los 2.000-2.100 m de altitud.

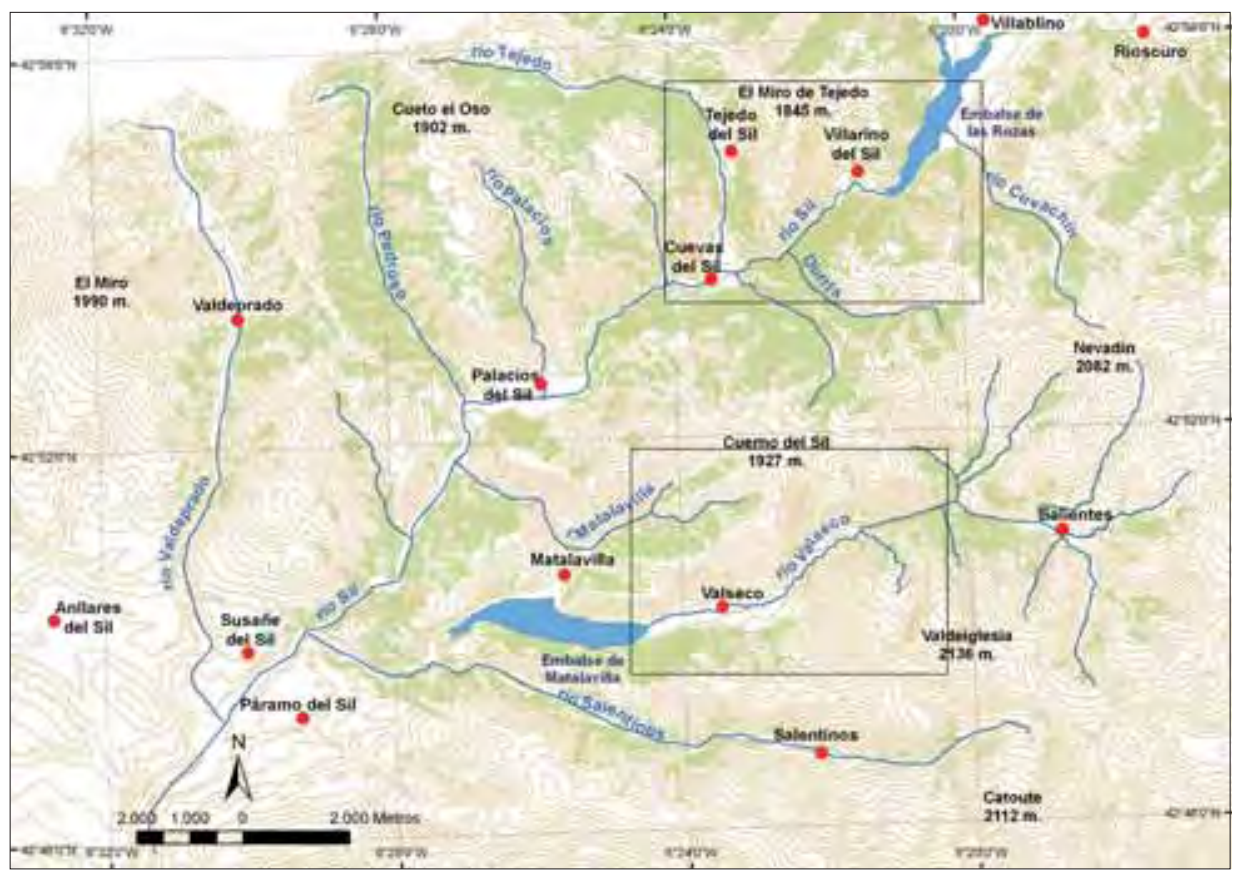

Figura 1. Mapa del área de estudio. Curvas de nivel cada 50 metros. Los cuadros indican las dos zonas para las que se presenta una cartografía específica. 
El clima de la zona es atlántico, aunque la influencia mediterránea es intensa en el extremo SW del Alto Sil. Las precipitaciones superan los $1.000 \mathrm{~mm}$ en todo el territorio y alcanzan valores superiores a los $2.000 \mathrm{~mm}$ a partir de unos $1.600 \mathrm{~m}$. Existe un máximo invernal muy marcado, que hace que gran parte de las precipitaciones sean en forma de nieve, siendo habitual que haya entre una y dos nevadas anuales con precipitaciones superiores a $30 \mathrm{~mm}$ en 72 horas a partir de sólo $1.100 \mathrm{~m}$ de altitud (Fig. 2). Estimamos que se pueden superar 4 eventos anuales a partir de $1.400 \mathrm{~m}$, si bien no existen datos dada la escasez de observatorios en la zona. Son estos episodios de nevadas copiosas, especialmente si están acompañadas de vientos que redistribuyen la nieve acumulándola en determinadas zonas, los que pueden producir aludes, observándose una reducción de su frecuencia en los últimos años.

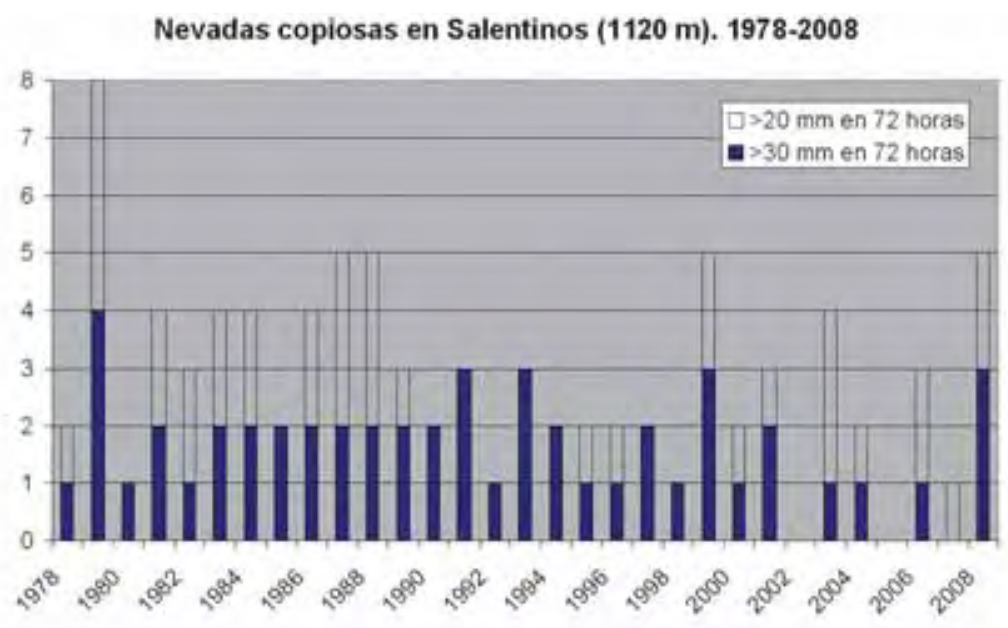

Figura 2. Precipitaciones en forma de nieve superiores a $20 \mathrm{~mm}$ y a $30 \mathrm{~mm}$ en Salentinos $(1.120 \mathrm{~m})$, entre los años 1978 y 2008.

El Alto Sil es un territorio boscoso, con unas 6.500 hectáreas ocupadas por frondosas (abedulares, robledales atlánticos, rebollares, bosques mixtos y, en el extremo suroeste, encinares y castañares). En total, suponen en torno al 19\% del área de estudio. En ellos habitan numerosas especies protegidas por diversas directivas europeas y extinta en gran parte del continente, entre la que sobresalen el oso pardo (Ursus arctos) y el urogallo cantábrico (Tetrao urogallus subsp. cantabricus). Toda la zona está declarada LIC, ZEPA y parte de ella es, además, Reserva Mundial de la Biosfera.

Durante la última glaciación, este territorio estuvo ocupado por extensos glaciares de valle que discurrieron por prácticamente la totalidad de las arterias fluviales preglaciares, generando uno de los mayores sistemas glaciares peninsulares, integrados por el glaciar del Sil y de sus afluentes (García de Celis y Martínez Fernández, 2002; Redondo Vega, 2002; Redondo Vega et al., 2006). Los depósitos más bajos y de mayor interés, se sitúan en torno a Páramo del Sil y Susañe del Sil, entre 760 y 1.000 m de altitud (Santos 
González et al., 2006). Destacan también el conjunto de glaciares rocosos relictos desarrollados bajo los escarpes cuarcíticos de la Serie de los Cabos, paleozoica, que suponen el mejor ejemplo de estas formas en toda la Cordillera Cantábrica (Redondo Vega et al., 2004; Fernández Martínez y Fuertes Gutiérrez, 2009).

\section{Metodología}

El avance en el conocimiento del relieve terrestre y la aparición de los Sistemas de Información Geográfica (SIG) han generado una herramienta básica para los estudios geomorfológicos. Desde hace ya varios años, esta herramienta se ha aplicado en la realización de cartografía geomorfológica y, en particular, de cartografía de aludes, puesto que éstos suelen aparecer en áreas con unas características de orientación, altitud e inclinación determinadas, que difieren sólo levemente en función del territorio analizado (Furdada et al., 1995; Misir y Karahalil, 2002; Oberoi y Thakur, 2004).

Para el presente trabajo se ha recopilado información entre los años 2002 y 2009 de los ádenes producidos en el Alto Sil mediante observaciones directas en el campo, complementadas con la realización de encuestas en distintas localidades de la zona, lo que ha permitido conocer también datos de eventos históricos. Todo ello ha servido para establecer las zonas donde los ádenes son y han sido más frecuentes. Evidentemente, la cantidad de datos es superior en las zonas cercanas a los pueblos y a las principales infraestructuras, por lo que avalanchas en zonas poco accesibles han podido pasar inadvertidas.

Por otra parte, mediante el software ArcGIS 9.2 (ESRI®) se ha realizado un análisis de orientación, pendientes y altitud, a partir de un modelo digital de terreno (malla de resolución de 25 x 25 metros), con el fin de observar las zonas que reúnen características apropiadas para el desencadenamiento de avalanchas de nieve y compararlas con los datos reales. Además, se ha incluido información sobre la presencia o ausencia de bosque, entendiendo como tal cualquier masa forestal densa de porte arbóreo. La cartografía de las zonas boscosas se ha realizado a partir de ortofotografías digitales de la Junta de Castilla y León, del año 2008.

Una vez obtenidas las zonas que reúnen unas características de altitud, orientación, pendiente y vegetación adecuadas, se ha realizado una corrección de éstas en función de los datos obtenidos en el campo y de la morfología concreta de las laderas expuestas a este fenómeno. Para este trabajo se han seleccionado dos zonas en las que los aludes son más frecuentes y tienen mayores dimensiones, además de afectar a diversas infraestructuras (carreteras, ferrocarril, tendidos eléctricos), como son los entornos de Valseco y Villarino del Sil.

\section{Evolución reciente de los ádenes en el Alto Sil}

\subsection{Orientaciones}

La mayor parte de los aludes desencadenados durante los últimos años en el Alto Sil se ha generado en laderas con orientación E, SE o S, siendo muy escasos los localizados 
con orientación norte, salvo pequeños aludes de placa en los circos glaciares. Esa misma tendencia, aunque menos marcada, parece desprenderse de los datos ofrecidos por Palomo et al. (2008) en los Pirineos centrales y por Oller et al. (2006) en el Pirineo catalán, así como en otras montañas con radiación solar elevada (Misir y Karahalil, 2002).

Las razones que explican la mayor frecuencia de aludes en estas orientaciones parecen ser diversas. Por una parte, las laderas orientadas al norte suelen tener una cubierta forestal más extensa y densa (Tabla 1) lo que, como veremos más adelante, obstaculiza en gran medida el desencadenamiento de aludes.

Por otra parte, las laderas expuestas al sur presentan mayor radiación solar, lo que se traduce en frecuentes períodos de hielo/deshielo que generen líneas de debilidad más marcadas que favorecen la aparición de aludes, como se ha observado en algunas zonas del interior de Estados Unidos (Cooperstein et al., 2004). Esta frecuencia de períodos de hielo/deshielo en orientaciones sur explica también por qué, una vez asentado el manto nival, el suelo se congela en orientaciones Norte, mientras que no lo hace en laderas orientadas a mediodía, dando como resultado una mayor estabilidad de las primeras (Santos González et al., 2009).

Además, los aludes se producen en zonas donde hay una fuerte acumulación de nieve, por lo que la relación con los vientos dominantes es fundamental (McClung y Schaerer, 2006). En el Alto Sil, las precipitaciones de nieve normalmente vienen asociadas a vientos del oeste-suroeste o bien de norte-noroeste, lo que explica que los aludes sean mucho más frecuentes en orientaciones Este que Oeste, ya que las segundas raramente mantienen un espesor de nieve importante, mientras que en las primeras se pueden acumular volúmenes de nieve muy superiores a los que realmente han precipitado, gracias al efectivo transporte del viento (Mases et al., 1998; Corripio et al., 2004).

Un último factor destacable es el relieve derivado de la acción glaciar, más nítido en zonas con orientación norte, lo que se traduce en rupturas de pendiente más frecuentes y un relieve menos propicio para la aparición de aludes de grandes dimensiones.

\subsection{Pendientes}

Aunque los valores señalados como más propensos para la aparición de avalanchas varían según autores, en general, se acepta que se dan en laderas con pendientes de entre $28^{\circ}$ y $45^{\circ}$ de inclinación. Por encima de ese valor, raramente se pueden acumular espesores suficientes para la aparición de aludes, mientras que por debajo la nieve se suele mantener estable.

Por otra parte, la zona de tránsito de los aludes puede tener pendientes de hasta unos $15^{\circ}$, deteniéndose generalmente cuando la ladera tiene menor inclinación, aunque puede variar en función de las características del terreno y de las dimensiones del alud. En ocasiones, los aludes pueden llegar incluso a remontar parte de la ladera opuesta en sectores de valle muy angostos, como sucedió en los aludes de nieve de La Fastirona, en diciembre de 2008 (Fig. 3). 
Tabla 1. Superficie según pendientes y orientaciones en el Alto Sil. Las diferencias son escasas entre todas las orientaciones, aunque hacia el sur y suroeste las pendientes tienden a ser algo más suaves y la cubierta forestal es más escasa.

\begin{tabular}{|l|c|c|c|c|c|c|c|}
\hline Orientación & $\begin{array}{c}\text { Superficie } \\
\text { (ha.) }\end{array}$ & $\mathbf{\%}$ & $\mathbf{0 - 1 5}$ & $\mathbf{1 5 - 2 8}^{\circ}$ & $\mathbf{2 8 - 4 5 ^ { \circ }}$ & $\mathbf{> 4 5}^{\circ}$ & $\begin{array}{c}\text { \% de } \\
\text { bosque }\end{array}$ \\
\hline Llano & 256 & 0,7 & - & - & - & - & 0 \\
\hline Norte & 4.750 & 13,6 & 16,7 & 47,7 & 35,0 & 0,6 & 22,6 \\
\hline Nordeste & 3.697 & 10,6 & 17,0 & 47,4 & 34,8 & 0,7 & 18,0 \\
\hline Este & 3.879 & 11,1 & 14,3 & 46,4 & 38,7 & 0,7 & 17,5 \\
\hline Sudeste & 3.947 & 11,3 & 17,3 & 43,3 & 38,1 & 1,3 & 12,8 \\
\hline Sur & 4.910 & 14,1 & 20,4 & 45,9 & 33,1 & 0,6 & 15,5 \\
\hline Suroeste & 4.915 & 14,1 & 22,6 & 46,5 & 30,3 & 0,6 & 21,2 \\
\hline Oeste & 3.997 & 11,5 & 18,8 & 44,8 & 35,8 & 0,6 & 21,7 \\
\hline Noroeste & 4.584 & 13,1 & 17,1 & 41,8 & 39,7 & 1,4 & 22,5 \\
\hline TOTAL & 34.935 & 100 & 18,1 & 45,2 & 35,3 & 0,8 & 18,9 \\
\hline
\end{tabular}

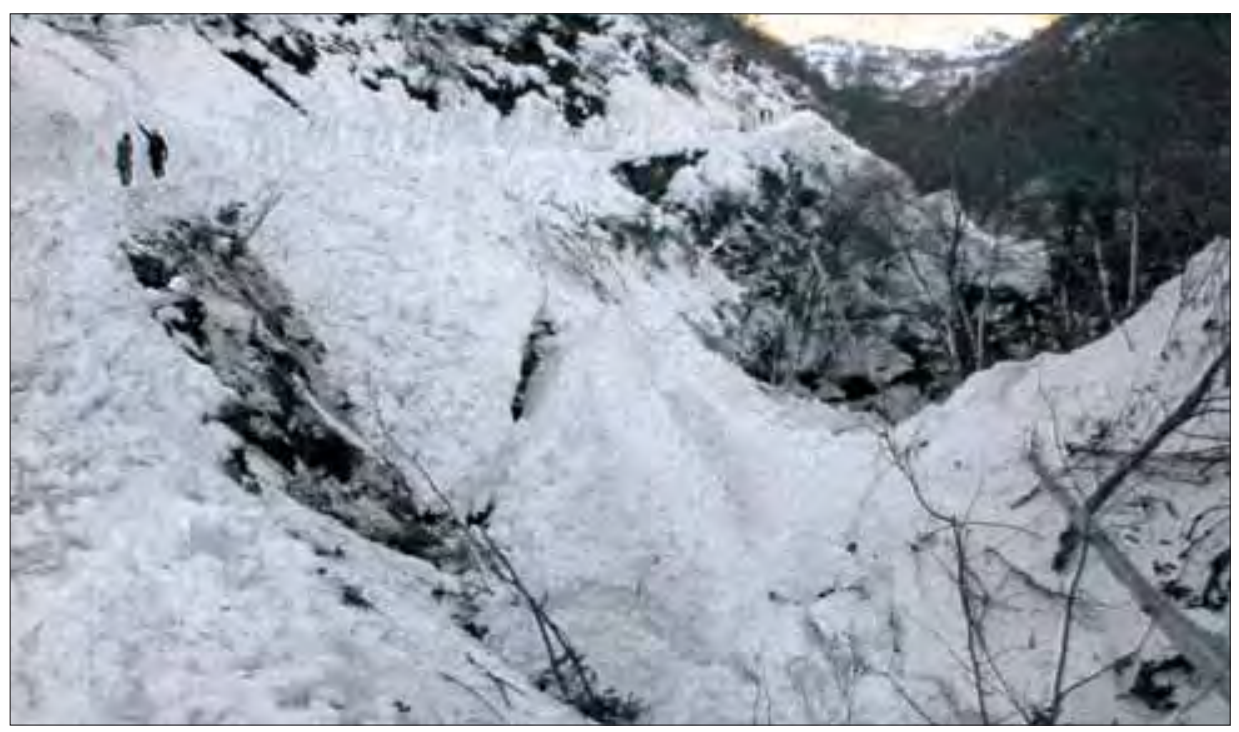

Figura 3. Alud de fondo en El Calzadón, en la carretera de acceso a Salientes. El río Valseco discurre bajo la nieve en la parte derecha de la fotografía. Nótese la presencia del guardarraíl en la ladera opuesta de la carretera y la gran cantidad de árboles derribados. Diciembre de 2008. 
La historia geológica del Alto Sil, muy influida por procesos de captura fluviales, hace que el relieve sea más enérgico en los tramos medios y bajos de los valles que en las áreas cimeras, caracterizadas por un relieve más suave aunque intensamente modelado por la acción glaciar. Todo ello hace que las pendientes medias sean más elevadas entre 1.000 y $1.400 \mathrm{~m}$, área en la que se desarrollan buena parte de los aludes de mayor significación.

En conjunto, más de una tercera parte de la superficie del Alto Sil tiene pendientes adecuadas para la aparición de aludes ya que, aunque no es un territorio caracterizado por pendientes muy fuertes (menos de un $1 \%$ tiene más de $45^{\circ}$ ), sí presenta en buena parte del territorio pendientes moderadas, especialmente por debajo de los $1.600 \mathrm{~m}$ (Tabla 2).

Tabla 2. Pendientes según rangos altitudinales en el Alto Sil. Destaca la extensa superficie existente entre 1.400 y $1.800 \mathrm{~m}$, a pesar de que sólo algunas cumbres superan los $2.000 \mathrm{~m}$ de altitud, así como que las pendientes más acusadas se sitúan preferentemente entre 1.000 y $1.400 \mathrm{~m}$.

\begin{tabular}{|l|c|c|c|c|c|c|c|c|}
\hline $\begin{array}{l}\text { Altitud } \\
\text { (m.s.n.m.) }\end{array}$ & $\begin{array}{c}\text { Superficie } \\
\text { (ha.) }\end{array}$ & $\mathbf{\%}$ & Llano & $\mathbf{0 - 1 5}^{\circ}$ & $\mathbf{1 5 - 2 8}^{\circ}$ & $\mathbf{2 8 - 4 5}^{\circ}$ & $\mathbf{> 4 5}^{\circ}$ & $\begin{array}{c}\mathbf{\%} \mathbf{d e} \\
\text { bosque }\end{array}$ \\
\hline$<1000$ & 4717 & 13,5 & 5,4 & 50,3 & 26,3 & 15,1 & 0,5 & 17,4 \\
\hline $1000-1200$ & 5582 & 16,0 & $<0,1$ & 17,7 & 38,8 & 40,8 & 1,1 & 26,1 \\
\hline $1200-1400$ & 6607 & 18,9 & $<0,1$ & 9,8 & 38,6 & 47,9 & 1,4 & 28,5 \\
\hline $1400-1600$ & 8119 & 23,2 & $<0,1$ & 10,9 & 50,2 & 36,7 & 0,6 & 21,3 \\
\hline $1600-1800$ & 6750 & 19,3 & $<0,1$ & 12,2 & 55,0 & 30,7 & 0,3 & 9,2 \\
\hline $1800-2000$ & 2982 & 8,5 & $<0,1$ & 15,0 & 56,1 & 27,1 & 0,4 & 0,8 \\
\hline$>2000$ & 177 & 0,5 & 0 & 18,5 & 43,3 & 36,7 & 1,5 & 0,0 \\
\hline Total & 34.935 & 100 & 0,7 & 17,7 & 44,3 & 34,6 & 0,8 & 18,7 \\
\hline
\end{tabular}

\subsection{Altitudes y topografía}

La altitud es un factor importante en el desencadenamiento de aludes, sobre todo porque influye notablemente en la aparición y persistencia del manto nival. Aunque en general son más frecuentes en las cotas más elevadas, por encima de los $1.800 \mathrm{~m}$, el relieve suave que define a las áreas cimeras de estas montañas y la intensa acción glaciar que las modeló, hace que raramente sobrepasen los 100 metros de longitud, frenándose al llegar al fondo de los circos glaciares. 
Además, la mayor parte de los aludes producidos a cotas altas son de placa, con efectos geomorfológicos muy limitados. Únicamente en algunas canales más desarrolladas, como en las Peñas del Infierno (entorno del Catoute, Salentinos) alcanzan una mayor significación. En estas zonas elevadas, son más frecuentes en los meses de marzo y abril, al contrario de lo que ocurre en cotas bajas, donde son más habituales entre diciembre y febrero.

En cualquier caso, es entre 1.200 y $1.600 \mathrm{~m}$ donde los aludes suelen alcanzar mayores dimensiones, puesto que es en esa zona donde las laderas presentan pendientes elevadas de forma más continua, especialmente en orientaciones meridionales, donde el modelado glaciar es menos nítido y existen menos rupturas de pendiente. Por otra parte, y aunque las nevadas son menos frecuentes que en cotas altas, la existencia de temperaturas más elevadas tras las nevadas, a veces con lluvia, se traduce en una mayor inestabilidad del manto nivoso, lo que facilita el desencadenamiento de aludes.

Destaca la baja altitud a la que pueden arrancar algunos aludes en esta zona, como en El Calzadón, que se inició a sólo 1.250 m de altitud, teniendo en cuenta que por ejemplo en el Pirineo las zonas de arranque de los aludes en los últimos 20 años no han descendido de $1.360 \mathrm{~m}$ (Oller et al., 2006).

\subsection{El papel del bosque}

La importancia de la cubierta vegetal para el control de aludes es clara y ha sido destacada por numerosos autores. En algunas zonas de Estados Unidos, se observó a lo largo del siglo XX una fuerte reducción de avalanchas en las zonas colonizadas por el bosque, mientras que en sectores deforestados por los incendios su frecuencia aumentaba (Reardon y Fagre, 2004). En otras zonas, como Turquía (Misir y Karahalil, 2002) o Canadá (Anderson y McClung, 2004) se destaca la importancia del bosque como factor de control para los aludes y, en Andorra y otros sectores del Pirineo, se potencia como un elemento protector frente a ellos (Solé et al., 2008).

En el Alto Sil, al igual que en el resto de la Cordillera Cantábrica, el bosque ha tenido una fuerte expansión desde mediados del siglo XX por el progresivo abandono del medio rural y el cambio en los usos del suelo. Esto ha hecho que muchas laderas que estaban ocupadas por pastizales hoy en día estén cubiertas de densos bosques, lo que se traduce en una reducción del espacio con riesgo de avalanchas.

Así, existen lugares donde se citan aludes a mediados del siglo XX que hoy en día están cubiertos de bosques maduros que imposibilitan su desencadenamiento, como ocurre entre Valseco y Salientes, o en la denominada Vallina del Ádene, en Salentinos. De hecho, los lugares con mayor riesgo actual del Alto Sil se sitúan en laderas afectadas por incendios forestales en los últimos 10-15 años, donde la cubierta forestal es escasa. Ello no impide que algunas avalanchas producidas en cotas altas destruyan árboles a su paso, como ha sucedido en todos aquellos de grandes dimensiones observados estos años. 
En conjunto, el bosque ocupa un $25 \%$ de las pendientes de entre $28^{\circ}$ y $45^{\circ}$, lo cual supone que una cuarta parte del terreno susceptible de iniciar aludes no lo hace, o sólo ocasionalmente, por la presencia de estas masas forestales, implicando una reducción significativa de la superficie afectada. Así, dentro de bosques, sólo hemos observado algunos aludes canalizados por los cauces de pequeños barrancos de fuerte pendiente en el entorno de Villarino del Sil.

En algunos casos, el bosque ocupa posiciones muy favorables para la protección de pueblos, como ocurre en algunas casas de Mataotero y en Salentinos, donde es posible que el mantenimiento tradicional de un espacio forestal en las laderas situadas por encima de estas localidades se deba, al menos en parte, a la protección que ofrecían frente a los ádenes.

\section{Cartografía de zonas de aludes en el Alto Sil}

A partir de la observación del territorio, de los aludes producidos entre 2002 y 2009, de entrevistas a la población local y de las referencias existentes sobre este fenómeno, se han definido cuatro categorías de riesgo para zonas de salida de los aludes, en función de sus características y la probabilidad de que sucedan (Fig. 4):

1. Zonas de riesgo alto. Se trata de áreas donde los ádenes han sido observados en varias ocasiones en los últimos años y que reúnen las siguientes características: altitud superior a $1.400 \mathrm{~m}$, pendientes de entre $28^{\circ}$ y $45^{\circ}$, orientaciones entre $90^{\circ}$ y $202^{\circ}$. El mayor riesgo se da cuando estas zonas coinciden, además, con canales de aludes capaces de transportar la nieve a cotas bajas.

2. Zonas de riesgo medio. Son aquellas donde los aludes se pueden producir de forma ocasional y agrupan dos tipos de localizaciones:

- Altitud superior a $1.400 \mathrm{~m}$, pendientes de entre $28^{\circ}$ y $45^{\circ}$, orientación entre $337^{\circ}$ y $90^{\circ}$ (NW, N y NE), o

- Altitud de entre 900 y $1.400 \mathrm{~m}$, con pendientes de entre $28^{\circ}$ y $45^{\circ}$, con orientación entre $90^{\circ}$ y $202^{\circ}$.

3. Zonas de riesgo bajo. Zonas situadas entre 1.000 y $1.400 \mathrm{~m}$ con pendientes de entre $28^{\circ}$ y $35^{\circ}$, con orientación entre $90^{\circ}$ y $202^{\circ}$, o bien con pendientes de entre $35^{\circ}$ y $45^{\circ}$ y cualquier orientación. En estas áreas no se han observado aludes en los últimos años pero pueden producirse si se dan unas condiciones apropiadas.

4. Zonas de riesgo de aludes en circos. Zonas situadas dentro de circos glaciares, a más de $1.800 \mathrm{~m}$, con pendientes de entre $28^{\circ}$ y $45^{\circ}$, con cualquier orientación. En casi todos los casos sólo se pueden producir aludes de pequeñas dimensiones, dado que las pendientes adecuadas raramente se mantienen durante más de 50-150 metros. 


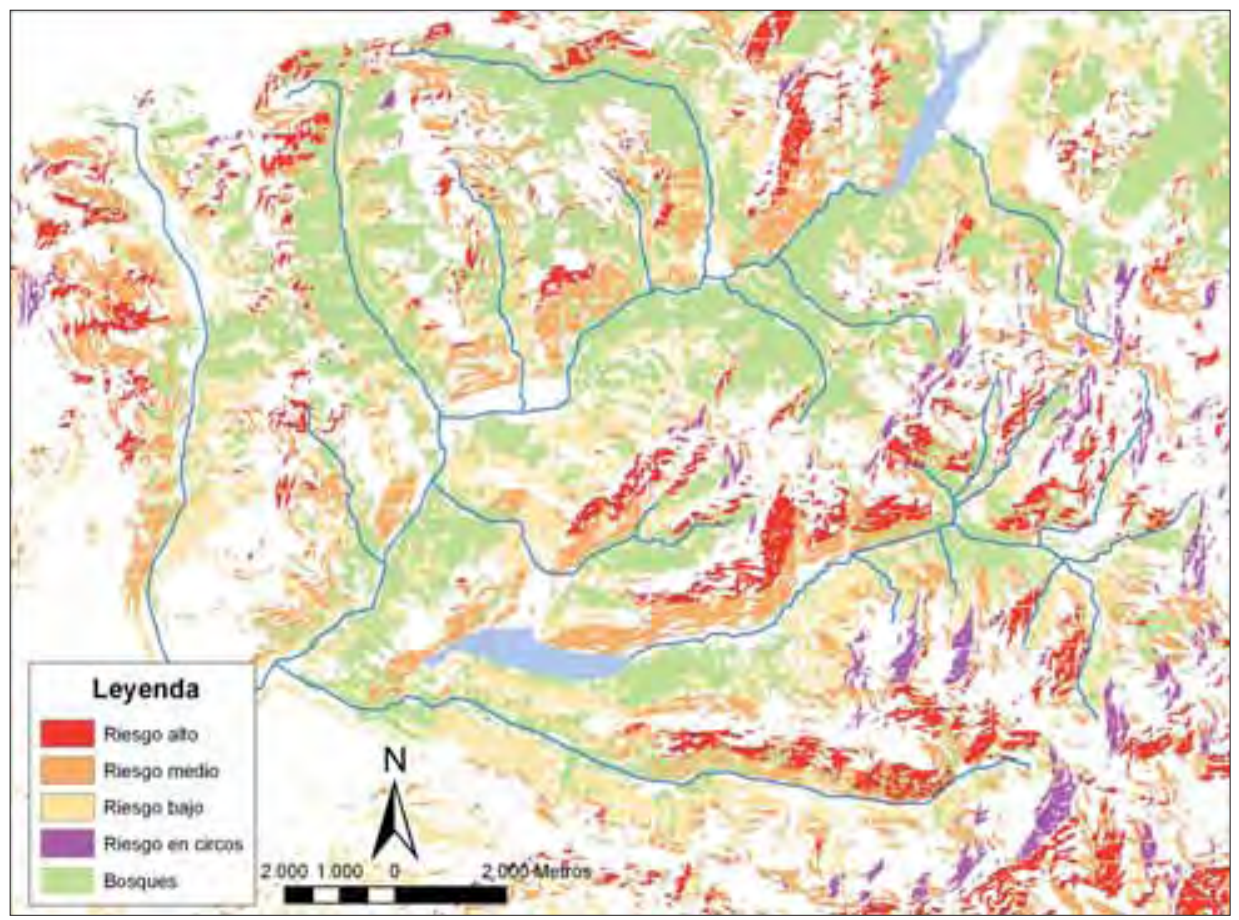

Figura 4. Zonas con riesgo de aludes en función de la orientación, pendiente media y altitud estimadas para el Alto Sil.

De todas formas, las dimensiones de los aludes que se desencadenan en estas zonas dependen de las características de las laderas por debajo de estos puntos y, por lo tanto, deben ser analizadas de forma concreta. Así, aunque algunos sectores son propicios para que se produzcan aludes, la inexistencia de zonas de transporte hace que el riesgo de aludes descienda notablemente (Luckman, 1977). Los aludes de mayor tamaño se producen donde existen canales capaces de transportar la nieve hasta cotas más bajas, y donde la pendiente se mantiene por encima de los $15^{\circ}$ (Butler y Walsh, 1990), ya que en caso contrario éste se detiene.

Por ello, se ha realizado una adaptación de esa cartografía general a otra específica, más detallada y realista. Así, con toda la información de campo, encuestas y gabinete se ha realizado una cartografía de riesgo de aludes para el Alto Sil, de la que se presentan dos zonas en mayor detalle, en los entornos de Valseco y Villarino del Sil.

La simbología de la cartografía es similar a la utilizada por el Institut Cartogràfic de Catalunya, aunque se han introducido algunas variantes, adaptándola a las condiciones del área de estudio. En la cartografía final se incluyen cuatro zonas asociadas a aludes: 
Aludes documentados. Se refiere a avalanchas observadas en los últimos años, bien de forma directa, bien a través de informaciones de otras personas. Las copiosas nevadas producidas en febrero de 2006 y diciembre de 2008, junto con otras entre enero y abril de 2009 , provocaron varios aludes, lo que ha facilitado la realización de una cartografía bastante completa, puesto que éstos se suelen producir de forma recurrente en los mismos lugares. Así, varias personas señalaron cómo en estos eventos se produjeron ádenes en los lugares dónde eran habituales hacía 20 o 30 años.

CANAl DE Aludes INACTIVA. Son zonas donde se produjeron aludes históricos, pero que actualmente están colonizadas por vegetación densa, que dificulta la aparición de aludes, y que no han mostrado ningún signo de actividad en los últimos años. Estas áreas, en caso de verse afectadas por incendios forestales o elementos antrópicos podrían volverse activas, de ahí que se incluyan en la cartografía.

RIESGO DE ALUDES. Se incluyen en esta categoría zonas con unas características de pendiente, orientación y topografía muy propicias para el desencadenamiento de aludes. En general, son zonas situadas por encima de 1.400 m y con orientación E, SE o S, o bien por encima de $1.700 \mathrm{~m}$, en el entorno de los circos glaciares.

RIESGO BAJO DE ALUDES. Se trata de áreas con pendientes de entre $28^{\circ}$ y $45^{\circ}$ y que reúnen condiciones topográficas adecuadas para que se generen avalanchas en caso de nevadas muy copiosas o condiciones especialmente favorables, pero que sólo pueden ocurrir en períodos de retorno elevados.

\subsection{Los aludes en el entorno de Valseco}

Entre Valseco y Salientes, existe un sector especialmente favorable para los aludes de nieve, en la que se han observado varios en los últimos años. El de mayores dimensiones se registró en febrero de 2006 en la canal de la Turria (Fig. 5), con una longitud de $1.400 \mathrm{~m}$ y un desnivel de $620 \mathrm{~m}$, entre 1.670 y $1.050 \mathrm{~m}$.

Aunque lo habitual es que los aludes se frenen en el cono de deyección existente a la salida del barranco, en ese mes, tras $168 \mathrm{~mm}$ de nieve en dos nevadas consecutivas (10 días en total), y vientos del oeste que pudieron acumular más de tres metros en algunas de las canales de La Turria, un gran ádene fluyó por la canal y llegó a atravesar la carretera de acceso a Salientes, derribando una veintena de álamos temblones de unos 30 metros de altura que estaban junto al río Valseco, así como el guardarraíl de la carretera, que quedó arrancado en un tramo de unos 50 metros. Además de este alud principal, se produjeron otros en las canales laterales.

En ese mismo entorno se han producido varios aludes tanto en la canal principal como en las laterales en marzo de 2008 y diciembre de 2008 y es conocido en Valseco como uno de los lugares con mayor frecuencia de ádenes, si bien lo habitual es que no desciendan hasta cotas tan bajas, sino que tengan su frente dentro de la propia canal principal. Los numerosos árboles de poco porte derribados en los aludes recientes parecen 


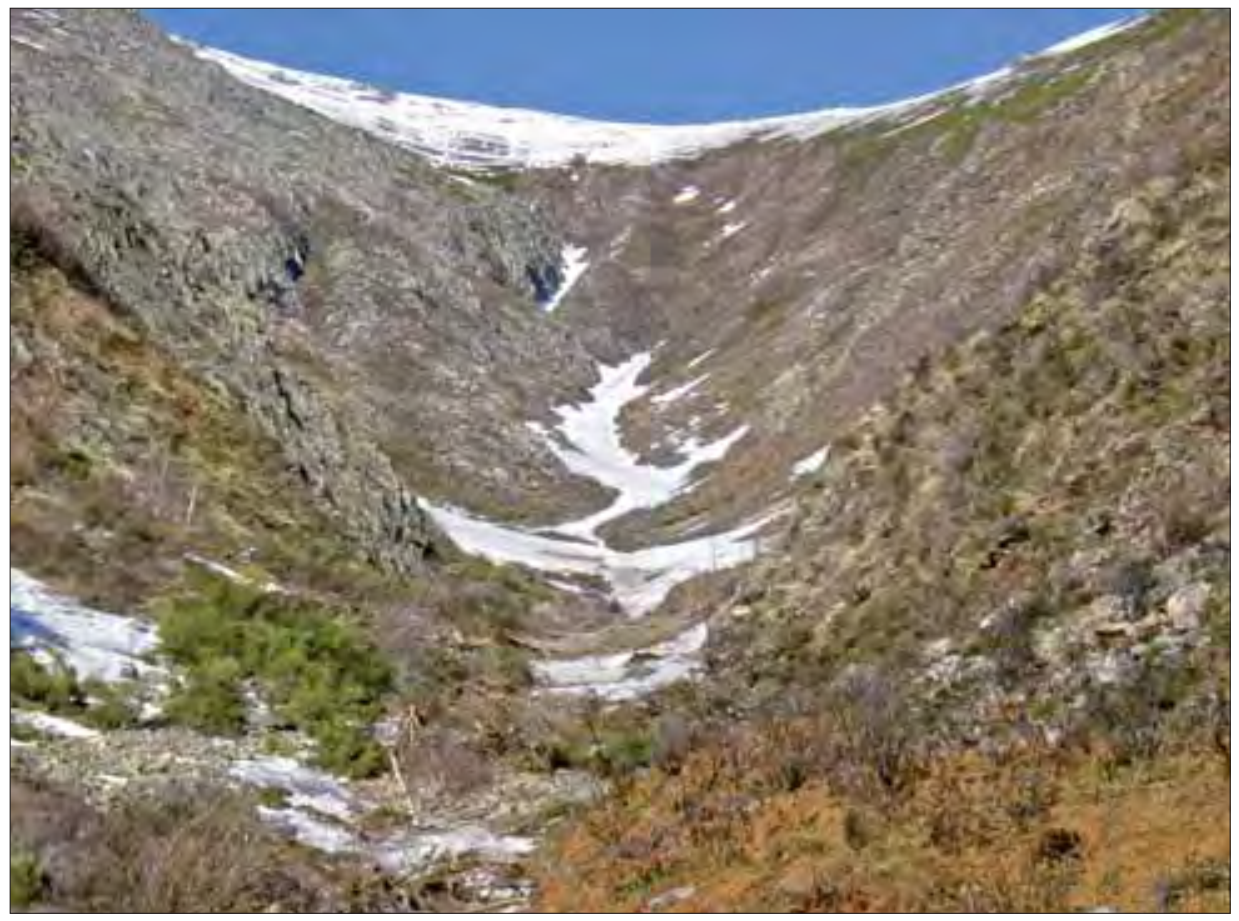

Figura 5. Vista del interior de la canal de La Turria, con restos de avalanchas de diciembre de 2008 y árboles derribados. En esta zona son frecuentes los aludes, en algunos casos laterales (desde la izquierda de la fotografía) y en otros canalizados por el barranco principal (zona frontal de la fotografía). Todo este sector ha sufrido varios incendios en los últimos años, lo que probablemente ha ayudado a la formación de ádenes.

indicar una falta de actividad en los últimos 10-15 años, coincidiendo con un período de escasez de grandes nevadas en la zona.

Otro alud significativo se produjo en El Calzadón (Fig. 6), tras una fuerte nevada en diciembre de 2008, que dejó 1,2 metros de espesor en 48 horas, sin que hubiera nieve previa en el suelo. Fue un ádene de ladera, de fondo y de nieve húmeda, bastante destructivo, que movilizó gran cantidad de pequeños robles y matorrales, arrancando también dos postes de teléfono, así como el guardarraíl de la carretera, que quedó empotrado contra la otra ladera, donde derribó numerosos alisos, abedules y fresnos. La gran masa de nieve y vegetación ocultó el río Valseco durante unas dos semanas, aunque el agua pudo circular por debajo de la nieve.

Trescientos metros al este de ese lugar, en los años 70 del pasado siglo se produjo un alud de grandes dimensiones en una pequeña canal hoy en día cubierta por abedules ya maduros. Según cuentan los vecinos de Salientes el ádene obligó a hacer un túnel en la nieve para poder circular, dada su gran magnitud, y provocó un gran represamiento 


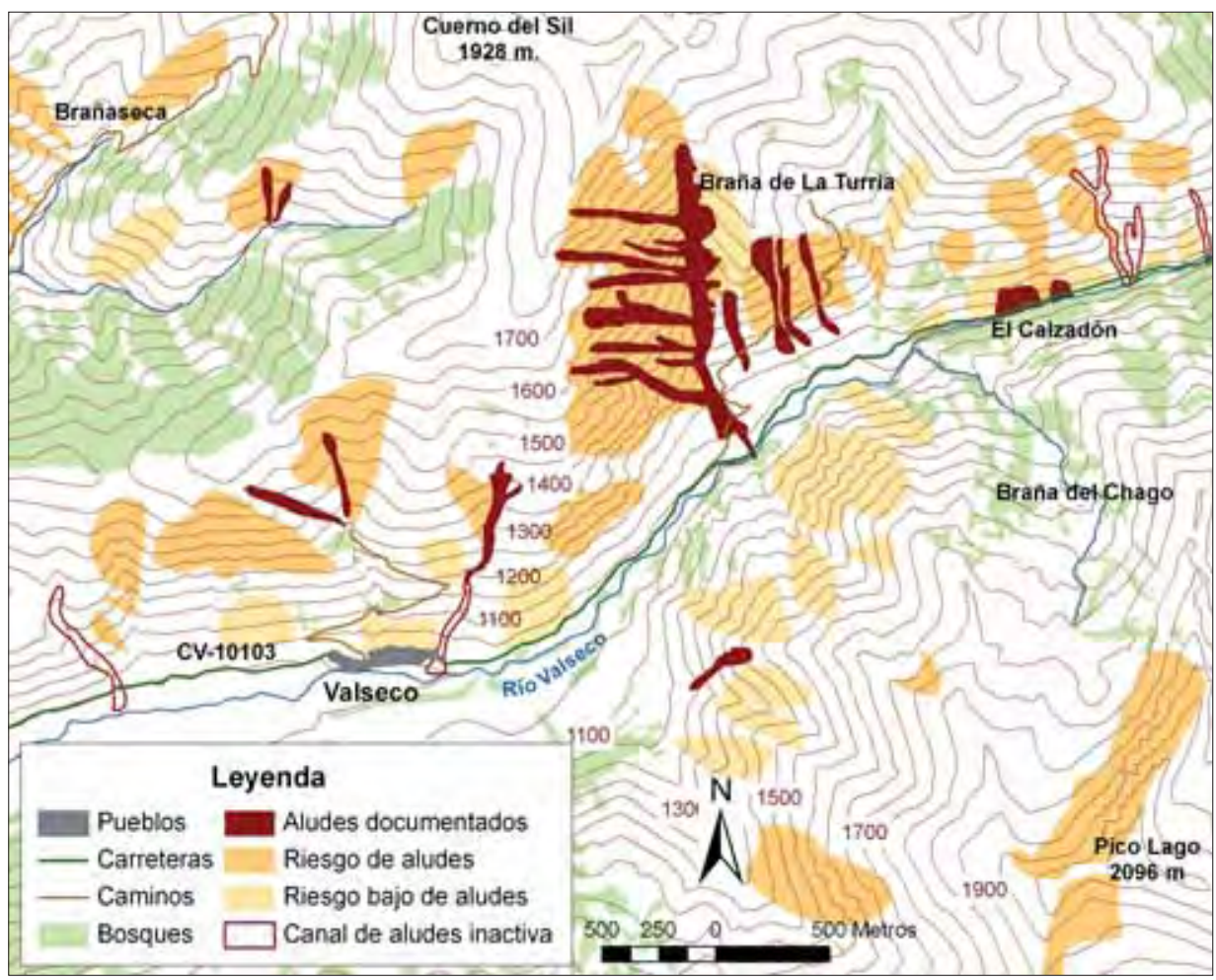

Figura 6. Cartografía de aludes en el área de Valseco (Alto Sil).

del río Valseco, que tardó varios días en horadar la nieve y poder discurrir de nuevo por su cauce. En ese mismo lugar, señalan que era frecuente que se produjeran aludes a mediados del siglo XX, aunque en los últimos 20 años no han tenido lugar, seguramente por la colonización vegetal que ha experimentado el entorno.

El pueblo de Valseco también se ha visto afectado históricamente por los aludes y, de hecho, según la tradición oral, antiguamente estaba formado por dos barrios, uno de ellos más alto, que fue despoblado debido a las avalanchas, que dañaban las casas, con lo que su relevancia debió de ser notable. La situación probable del barrio, inmediatamente al este de las últimas casas del pueblo, es una zona propensa para estos fenómenos tras grandes nevadas, justo a la salida de un profundo barranco orientado al sur y que arranca a unos $1.400 \mathrm{~m}$ de altitud.

Es fácil imaginar que por este pequeño barranco se hayan originado aludes de cierta importancia. En todo caso, también en el pueblo existe otro pequeño barranco que es susceptible de albergar ádenes, aunque la zona de reposo por encima del pueblo puede hacer que se detengan antes de las casas, por la menor pendiente, al situarse un cono de derrubios en esa zona. 


\subsection{Los aludes del entorno de Villarino del Sil}

Al oeste de Villarino del Sil, en el cordal meridional que se desgaja desde El Miro de Tejedo (Fig. 7), existen una serie de canales de aludes, orientadas al este y sureste, donde las avalanchas son especialmente frecuentes. En los últimos años las hemos observado en febrero de 2006, marzo de 2008, noviembre de 2008, diciembre de 2008 y en varias ocasiones en 2009, aunque parece que suelen desencadenarse casi todos los años. La existencia de una zona de relieve suave a barlovento ayuda a que la acumulación de nieve sea muy elevada en toda la zona y las pendientes, de en torno a $35^{\circ}$, junto con la presencia de barrancos que canalizan el drenaje, hacen que se creen unas condiciones especialmente favorables para los ádenes.

La altitud a la que se encuentran es variada, desde los $1.800 \mathrm{~m}$ que arrancan las canales más elevadas a los $1.450 \mathrm{~m}$ de las más bajas, teniendo un desnivel de entre 250 y $500 \mathrm{~m}$. La que presenta un mayor riesgo es la más meridional, puesto que su frente atraviesa la carretera C-631, muy cerca del cruce de Villarino (Fig. 8). A pesar de ser la menos activa y que el frente suele situarse a cotas superiores a la de esta vía de comunicación, a mediados de enero de 2009 un alud llegó a afectar a la carretera, sin que produjera daños significativos.

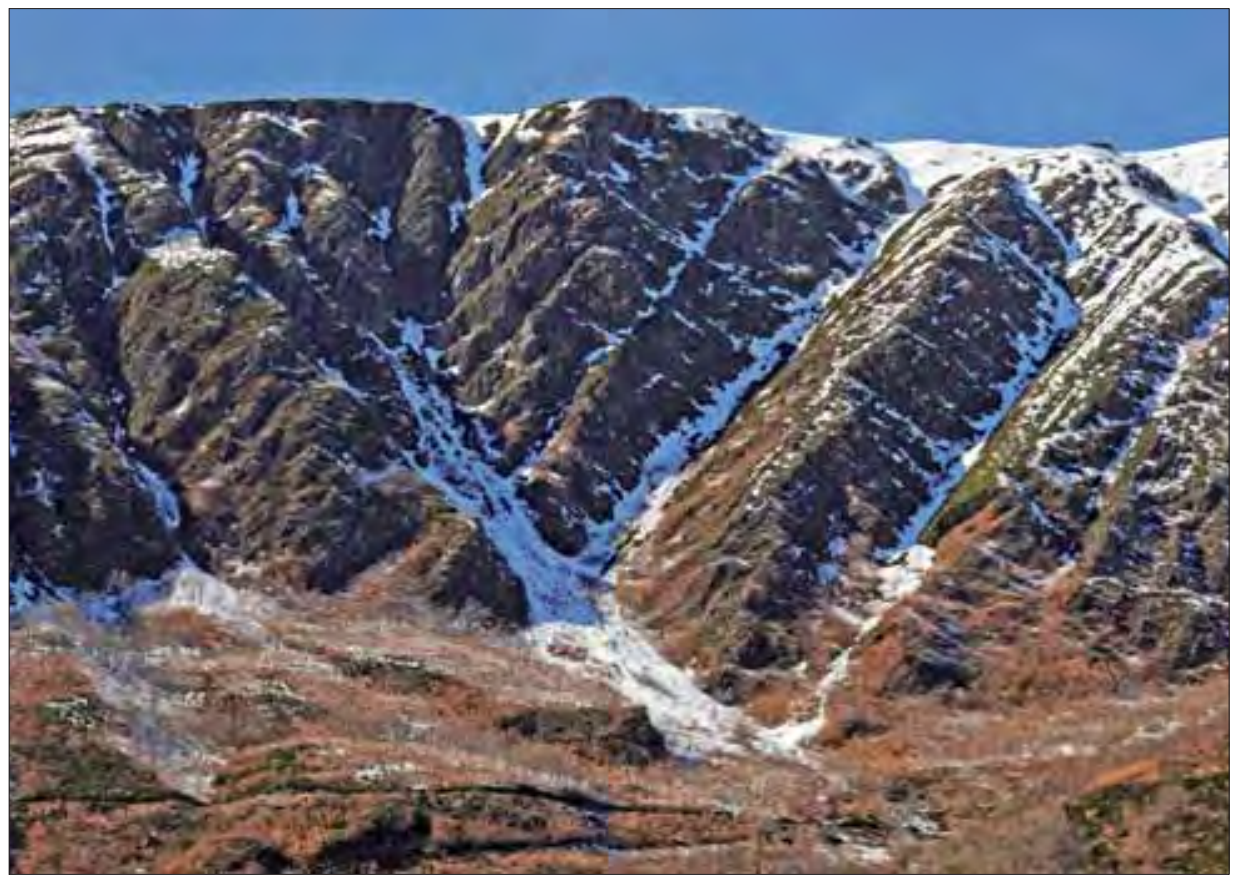

Figura 7. Vista de diversas canales de aludes en el entorno de Villarino del Sil.

La escasez de bosque al pie de las principales canales se explica por la recurrencia en la actividad de los aludes. 


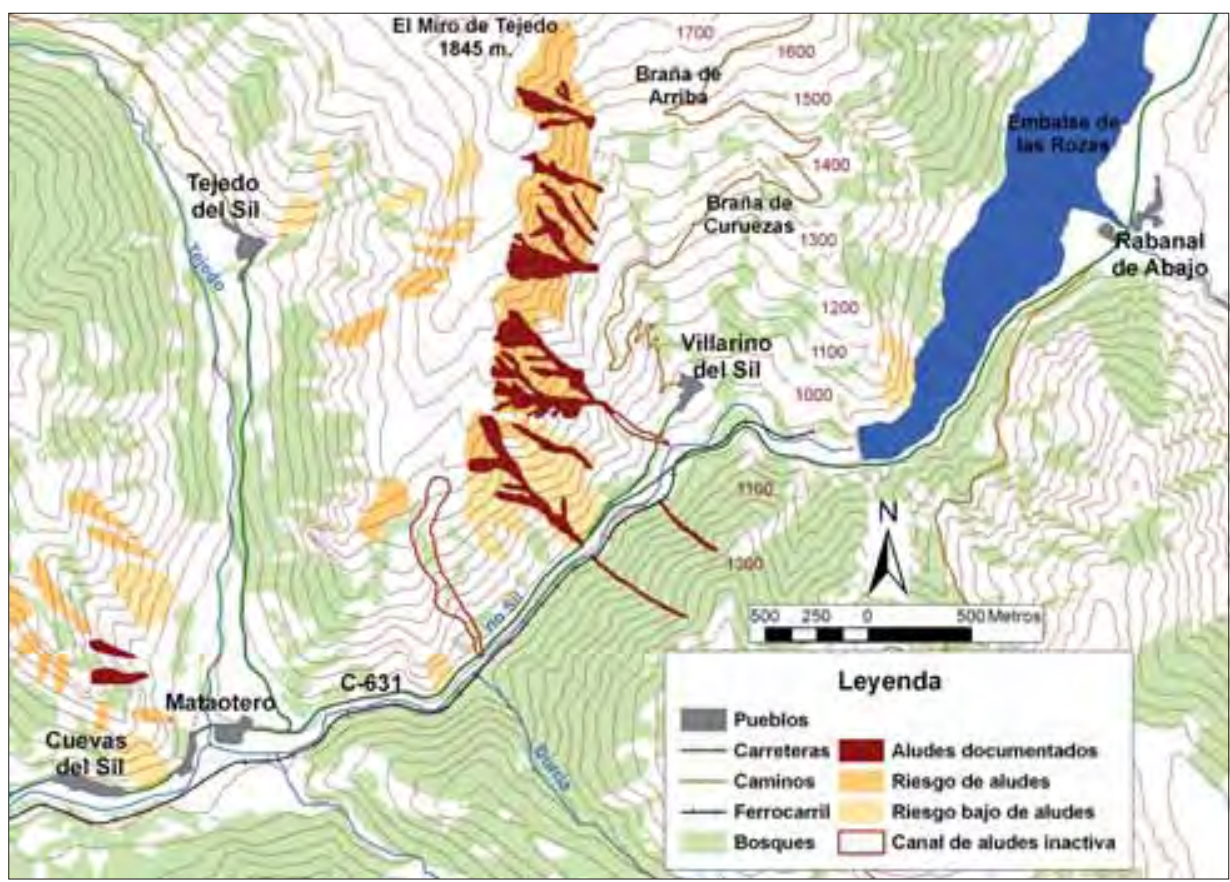

Figura 8. Cartografía de zonas de aludes en el entorno de Villarino del Sil.

En todas las canales del entorno de Villarino, especialmente en las meridionales, pueden observarse árboles arrancados por efecto de la nieve, que probablemente indican que la actividad de los aludes, tras años de nevadas muy escasas, ha aumentado en los años 2006, 2008 y, sobre todo, 2009, debido a las copiosas nevadas caídas en los meses invernales de estos años.

Fuera de ese entorno, los aludes también se producen, en este caso con orientación $\mathrm{NW}$, en algunos pequeños barrancos que drenan los extensos bosques mixtos de la margen izquierda del río Sil, entre Villarino y Cuevas del Sil (Fig. 8). En los últimos años hemos observado dos aludes en este sector, de carácter lineal y de tamaño reducido, ya que únicamente descienden siguiendo el cauce de pequeños arroyos de fuerte pendiente, dado que el bosque ejerce una labor protectora importante. Algunos de estos ádenes afectan al ferrocarril Ponferrada-Villablino, que discurre junto al río Sil. De hecho, según informaciones de algunos vecinos de la zona, el trazado de la línea se modificó para incluir varios pequeños túneles, debido a la frecuencia con la que las avalanchas afectaban al tránsito ferroviario.

\section{Conclusiones}

A partir del uso de los Sistemas de Información Geográfica, de las observaciones de campo y de la realización de encuestas a la población local, se ha realizado una car- 
tografía de aludes para el Alto Sil, de la que se presentan dos ejemplos de las zonas con una mayor actividad.

Las zonas con una mayor frecuencia en la aparición de aludes se sitúan entre 1.700 y $1.400 \mathrm{~m}$, con pendientes de entre $28^{\circ}$ y $45^{\circ}$ y con orientaciones SE y S, en zonas con escasa vegetación arbórea y, en la mayor parte de los casos, afectadas por incendios forestales en los últimos años.

Fuera de estos entornos, son también frecuentes en el ámbito de los circos glaciares, por encima de $1.800 \mathrm{~m}$ de altitud, si bien se trata generalmente de aludes de placa de pequeñas dimensiones y mucho menos peligrosos que los que se dan en cotas bajas, al igual que ocurre en otras zonas de la Cordillera Cantábrica (Castañón, 1984; González Gutiérrez, 2002).

Aunque la mayor parte de los ádenes se han producido en áreas sin usos humanos, algunos de ellos sí han afectado a infraestructuras, destacando los aludes producidos en la canal de La Turria en diciembre de 2006 y en El Calzadón en diciembre de 2008, ya que mantuvieron cortada la carretera de acceso a Salientes, manteniendo esta localidad aislada durante unas horas en el primer caso y 4 días en el segundo.

Consideramos que aunque los daños por aludes de nieve no son tan importantes en la Cordillera Cantábrica como los producidos en otras cadenas montañosas como los Alpes o los Pirineos, es necesario mejorar el conocimiento de este fenómeno con el objetivo de minimizar los daños y realizar una correcta gestión del territorio, teniendo en cuenta que suponen un riesgo natural, más aún si consideramos el creciente uso turístico que soportan estas montañas en los meses invernales.

\section{Agradecimientos}

Nuestro agradecimiento a diversas personas por la información aportada acerca de aludes producidos en el Alto Sil, en especial a Vitín, Manuel, Luterio, Gloria y Saúl, de Salientes, y a Senén, Amado y Fernando, de Salentinos.

Este trabajo ha sido posible gracias a una beca doctoral de la Universidad de León para la realización del trabajo titulado "Glaciarismo y periglaciarismo en el Alto Sil, provincia de León (Cordillera Cantábrica)". Así mismo, se enmarca dentro del proyecto "Dinámica geomorfológica en el entorno de los glaciares rocosos de la Cordillera Cantábrica" (LE020A07), financiado por la Junta de Castilla y León.

\section{Referencias bibliográficas}

Alcalde, M. (2002). La muelda de Huelde. Revista Comarcal Montaña de Riaño, 7: 5-6.

Anderson, G., McClung, D. (2004). Snow avalanche penetration into mature forest in timber harvested terrain. ISSW 2004 Preceedings. A merging of theory and practice. International Snow Science Workshop: 409-414, Jackson Hole (Wyoming). 
ButLer, D. R., WALSh, S. J. (1990). Lithologic, Structural, and Topographic Influences on Snow-Avalanche Path Location, Eastern Glacier National Park, Montana. Annals of the Association of American Geographers, 80 (3): 362-378.

Carcavilla, L., LóPeZ, J. (2000). Las avalanchas de nieve como riesgo natural. Terralia, 17: 46-54.

CAStañón, J. C. (1984). Sobre el modelado originado por los aludes de nieve en el Prau del Albo (Alto Huerna, Asturias). Ería, 6: 106-112.

Cooperstein, M. S., Birkeland, K. W., Hansen, K. J. (2004). The effects of slope aspect on the formation of surface hoar and diurnally recrystalized near-surface faceted crystals: implications for avalanche forecasting. ISSW 2004 Preceedings. A merging of theory and practice. International Snow Science Workshop: 83-93, Jackson Hole (Wyoming).

Corripio, J. G., Durand, Y., Guyomarc'H, G., Merindol, L., Lecorps, D., Pugliese, P. (2004). Land-based remote sensing of snow for the validation of a snow transport model. Cold Regions Science and Technology, 39: 93-104.

CruZ, J. (1987). Los aludes o avalanchas de nieve en España. Cuadernos de Protección Civil, 15-20.

Decaulne, A., Sæmundsson, D. (2006). Geomorphic evidence for present-day snowavalanche and debris-flow impact in the Icelandic Westfjords. Geomorphology, 80 (1-2): 80-93.

FERnÁNDEZ MiguÉlez, E. (2000). Dialecto leonés y el nombre de las cosas. Edilesa: 141 pp., León.

Fernández Martínez, E., Fuertes GutiérRez, I. (Coords.) (2009). Lugares de Interés Geológico. León. Junta de Castilla y León, DVD publicado por la Fundación Patrimonio Natural: ISBN 987-84-692-5657-2.

Furdada, G., (1996). Estudi de les allaus al Pirineu occidental de Catalunya: predicció espacial $i$ aplicacions de la cartografia. Geoforma Ediciones: 315 pp., Logroño.

Furdada, G., (2006). Aludes de nieve. Riesgo actual y riesgo futuro. Cuaternario y Geomorfología, 20 (3-4): 73-88.

Furdada, G., Martí, G., Oller, P., García, C., Mases, M., Vilaplana, J. M. (1995). Avalanche mapping and related G.I.S. applications in the Catalan Pyrenees. Surveys in Geophysics, 16: 681-693.

García de Celis, A., Martínez Fernández, L. C. (2002). Morfología glaciar de las montañas de la cuenca alta de los ríos Sil, Omaña, Luna y Bernesga: revisión y nuevos datos (Montaña Occidental de León). En: El modelado de origen glaciar en las montañas leonesas (Redondo, J. M., Gómez, A., González Gutiérrez, R. B. y Carrera, P., coords.). Universidad de León, pp. 137-196, León. 
GavaldÀ, J., Moner, I. (2008). Nou sistema gràfic de predicció local del perill d'allaus a la Val d'Aran. $3^{a}$ Jornada Tècnica de neu i allaus. Institut Geològic de Catalunya, Betren.

GonzÁlez Gutiérrez, R. B. (2002). El relieve de los valles del Torío y Curureño (Montaña Cantábrica Leonesa). Universidad de León: 267 pp., León.

González Trueba, J. J. (2006). El Macizo Central de los Picos de Europa: Geomorfología y sus implicaciones geoecológicas en la alta montaña cantábrica. Tesis doctoral. Departamento de Geografía, Urbanismo y Ordenación del Territorio, Universidad de Cantabria, Santander, 819 pp.

Julián, A., ChuecA, J. (1999). Cartografía de zonas probables de aludes en el valle de Ordesa (Pirineo aragonés). Geographicalia, 37: 73-86.

Julián, A., Peña, J. L., Chueca, J., Zabalza, J., Lapeña, A., López Moreno, I. (2000). Cartografía de zonas probables de aludes en el Pirineo aragonés: metodología y resultados. Boletín de la A.G.E., 30, 119-134.

Luckman, B. H. (1977). The Geomorphic Activity of Snow Avalanches. Geografiska Annaler, 59 A (1-2): 31-48.

Marquínez, J. L., Menéndez, R. A., Lastra, J., Fernández, E., Jiménez, B., Wozniak, E., Fernández, S., García, J., García, P., Álvarez, M. A., Lobo, T., Adrados, L. (2003). Riesgos naturales en Asturias. KRK Editores: 136 pp., Oviedo.

MARTín, R. (2003). Los aludes. Una revisión sobre sus características en las montañas españolas, y algunos modelos de prevención y protección. Revista de Protección Civil, 15: 8-10.

MASES, M. (2005). La nieve y los aludes. Actas de las Terceres Jornades del CRECIT. La didáctica dels riscos naturals. Institut d'Estudis Andorrans, Sant Julià de Lòria.

Mases, M., Vilaplana, J.M. (1991). Zonas de aludes en la Vallferrera: clasificación y riesgo geomorfológico. Pirineos, 138, 39-52.

Mases, M., Buisson, L., Frey, W., Martí, G. (1998). Empirical model for snowdrift distribution in avalanche-starting zones. Annals of Glaciology, 26: 445-449.

McClung, D. M., Schaerer, P. (2006). The Avalanche Handbook (3 ${ }^{\text {rd }}$ edition). Mountaineers Book: 288 pp., Seattle.

MisiR, M., KarahaliL, U. (2002). Determination of potential avalanche areas with GIS in Blacksea Region. International Symposium on GIS, Istanbul.

Oberoi, S. W., Thakur, N. K. (2004). Generation of disaster management model based on GIS and remote sensing. Proceedings of the Third International Conference on Theoretical, Applied, Computational and Experimental Mechanics (ICTACEM'04). Indian Institute of Technology Kharagpur: 295-298, Kharagpur. 
Oller, P., Muntán, E., Marturià, J., García, C., García, A., Martínez, P. (2006). The avalanche data in the Catalan Pyrenees. 20 years of avalanche mapping. Proceedings of the International Snow Science Workshop. 305-313, Colorado.

Palomo, M., Espejo, F., Ferraz, J. (2008). Análisis nivológico y climatológico del Refugio de Góriz (Huesca), 1981-2007. $3^{a}$ Jornada Tècnica de neu i allaus. Institut Geològic de Catalunya, Betren.

ReArdon, B. A., FAgre, D. B. (2004). Natural avalanches and transportation: a case study from Glacier National Park, Montana, USA. ISSW 2004 Preceedings. A merging of theory and practice. International Snow Science Workshop: 582-597, Jackson Hole (Wyoming).

REDOndo vegA, J. M. (2002). El relieve glaciar de la Sierra de Gistredo (NW de la Cordillera Cantábrica, León). En: El modelado de origen glaciar en las montañas leonesas (Redondo, J. M., Gómez, A., González Gutiérrez, R. B. y Carrera, P., Coords.). Universidad de León, pp. 105-136, León.

Redondo vega, J. M., Gómez villar, A., González Gutiérrez, R .B. (2004). Localización y caracterización morfométrica de los glaciares rocosos relictos de la Sierra de Gistredo (Montaña Cantábrica, León). Cuadernos de Investigación Geográfica, 30: $35-60$.

Redondo Vega, J. M., González Gutiérrez, R. B., Santos GonzÁlez, J., Gómez VILlaR, A. (2006). Sedimentación glaciolacustre en la Montaña Cantábrica Leonesa, pp. 83-100. En: Geomorfología y territorio. Actas de la IX Reunión Nacional de Geomorfología (Pérez Alberti, A y López Bedoya, J., Eds.). Universidade de Santiago de Compostela, pp. 83-100, Santiago de Compostela.

Santos González, J., Redondo Vega, J. M., Gómez Villar, A., González Gutiérrez, R. B. (2006). Bloques erráticos en Páramo del Sil: testigos del máximo avance glaciar en la cuenca del Sil (Cordillera Cantábrica), pp. 101-112. En: Geomorfología y territorio. Actas de la IX Reunión Nacional de Geomorfología (Pérez Alberti, A y López Bedoya, J., Eds.). Universidade de Santiago de Compostela, pp. 101-112, Santiago de Compostela.

Santos González, J., González Gutiérrez, R. B., Gómez Villar, A., Redondo VEGA, J. M. (2009). Ground thermal regime in the vicinity of relict rock glaciers (Cantabrian Mountains, NW Spain). Finisterra, 87: 35-44.

Solé, X., Domènech, M., Aunós, A. (2008). Prospecció per l'obtenció d'una metodologia per a la caracterització silvícola dels boscos protectors d'Andorra contra allaus. $3^{a}$ Jornada Tècnica de neu i allaus. Institut Geològic de Catalunya, Betren.

Wozniak, E., Marquínez, J. (2004). Evaluación de la susceptibilidad por aludes de nieve a escala regional: el caso de Asturias. En: Riesgos Naturales y Antrópicos en Geomorfología (Benito, G. y Díez Herrero. A., Eds.). Sociedad Española de Geomorfología y CSIC-Centro de Ciencias Medioambientales, pp. 509-518, Madrid. 